\title{
COLLAGEN FIBRILLOGENESIS IN THE DEVELOPMENT OF THE ANNULUS FIBROSUS OF THE INTERVERTEBRAL DISC
}

\author{
Anthony J. Hayes*, Marc D. Isaacs, C. Hughes, B. Caterson and J.R. Ralphs \\ Connective Tissue Biology Laboratory and Cardiff Institute of Tissue Engineering and Repair, Cardiff School of \\ Biosciences, Cardiff University, Cardiff CF10 3US, Wales, UK
}

\begin{abstract}
The annulus fibrosus of the intervertebral disc is a complex, radial-ply connective tissue consisting of concentric lamellae of oriented collagen. Whilst much is known of the structure of the mature annulus, less is known of how its complex collagenous architecture becomes established; an understanding of which could inform future repair/ regenerative strategies. Here, using a rat disc developmental series, we describe events in the establishment of the collagenous framework of the annulus at light and electron microscopic levels and examine the involvement of class I and II small leucine rich proteoglycans (SLRPs) in the matrix assembly process. We show that a period of sustained, ordered matrix deposition follows the initial cellular differentiation/orientation phase within the foetal disc. Fibrillar matrix is deposited from recesses within the plasma membrane into compartments of interstitial space within the outer annulus - the orientation of the secreted collagen reflecting the initial cellular orientation of the laminae. Medially, we demonstrate the development of a reinforcing 'cage' of collagen fibre bundles around the foetal nucleus pulpous. This derives from the fusion of collagen bundles between presumptive end-plate and inner annulus. By birth, the distinct collagenous architectures are established and the disc undergoes considerable enlargement to maturity. We show that fibromodulin plays a prominent role in foetal development of the annulus and its attachment to vertebral bodies. With the exception of keratocan, the other SLRPs appear associated more with cartilage development within the vertebral column, but all become more prominent within the disc during its growth and differentiation.
\end{abstract}

Keywords: Intervertebral Disc - Development, ECM Collagens, ECM - Proteoglycans, Imaging - EM, Imaging - LM.

*Address for correspondence:

Anthony J. Hayes,

Confocal Microscopy Unit,

School of Biosciences, Cardiff University

Cardiff, CF10 3AX, Wales, UK.

Telephone Number : +44(0)2920876611

E-mail: Hayesaj@cf.ac.uk

\section{Introduction}

The annulus fibrosus of the intervertebral disc (IVD) is a highly ordered, radial-ply, fibrous connective tissue. It consists of a nested series of circumferential coaxial lamellae that enclose the nucleus pulposus of the disc laterally, whilst uniting adjacent vertebral bodies via their endplates rostro-caudally (Peacock 1951a,b; Walmsley, 1953; Inoue and Takeda, 1975; Hashizume, 1980; Inoue, 1981). The lamellae are composed largely of collagen fibre bundles, with type I collagen predominating in the outermost annulus and type II collagen making up most of the inner annular lamellae (Eyre and Muir, 1977). Interspersed among these are other extracellular matrix (ECM) components including proteoglycans (PGs), glycoproteins, and elastic fibres, in addition to the connective tissue cells that secrete and organise these components (Hayes et al., 2001). Within individual lamellae, collagen fibre bundles are precisely arranged at a constant angle to the long axis of the vertebral column. The inclination of this angle alternates in successive lamellae, so that every other lamella has the same collagen fibre orientation (Peacock1951a,b; Walmsley, 1953; Hickey and Hukins, 1980; Hukins and Meakin, 2000). Connectivity between lamellae occurs via translamellar bridges (Melrose et al., 2008; Schollum et al., 2009), which may help maintain stability within the annulus. This organisation is of fundamental importance to IVD function: it endows the annulus radial-ply strength, allowing it to resist compressive loads exerted on it by the nucleus pulpous in response to gait and spinal muscle activity, whilst permitting limited torsion and flexion movements of the spine (reviewed by Bogduk, 2005). Whilst much is known of the structure and function of the annulus, our understanding of how its complex organisation is established during development is poor.

The initial organisation of the annulus is set up during early foetal development and appears to be triggered by expansion of the notochord (Aszódi et al. 1998). This occurs at embryonic day 55 in the human (Peacock, 1951a) and between embryonic days 15 and 16 in the rat (Rufai et al., 1995). Initially, mesenchymal cells surrounding the notochord form dense annular condensations separated by the cartilage of the primordial vertebral bodies. As the vertebral bodies undergo chondrogenesis, the notochord bulges rapidly in the region of the developing IVD thus giving rise to the foetal nucleus pulposus. At the same time the intervertebral cell condensations differentiate into the annulus fibrosus. The inner aspect of the nascent annulus is cartilaginous; whereas the outer annulus consists of highly oriented fibroblastic laminae, whose cellular organisation precedes the later lamellar organisation of 
oriented collagen (Peacock, 1951a; Rufai et al., 1995). Underlying this initial cellular template is an integrated network of oriented actin stress-fibres coupled via adherens junctions (Hayes et al., 1999). With the onset of matrix synthesis, cell-ECM adherens junctions and integrins such as $\alpha 5 \beta 1$ then appear important in the organisation of oriented collagenous lamellae (Hayes et al., 1999). The cellular mechanisms underlying the formation of the annular lamellae are not understood; however, they may be similar to those occurring in other oriented connective tissues. In tendon and cornea, for example, collagen fibrils are self assembled at recesses within the cell membrane; their growth into fibres, bundles and fascicles/lamellae then appears to occur via a series of extracellular compartments elaborated by the cells (Birk and Trelstad, 1984, 1986; Canty and Kadler, 2002; Canty et al., 2004; Banos et al., 2008). The precise orientation and organisation of collagen fibres within these structures must be tightly controlled with high fidelity for coordinated growth of the tissue as a whole. This control is provided by a range of ECM molecules (reviewed by Banos et al., 2008) that include the SLRPs (small leucine rich proteoglycans), FACIT collagens (fibril-associated collagens with interrupted triple helices) such as collagen types XII and XIV, and other ECM molecules including COMP (Cartilage oligomeric matrix protein).

The SLRPs, in particular, function as key regulators of ECM assembly and tissue growth in oriented fibrous connective tissues (Banos et al., 2008; Kalamajski and Oldberg, 2010). Family members are grouped into five distinct classes that are all characterised by the presence of a leucine rich repeat, a highly variable $\mathrm{N}$-terminal domain which may have attached glycosaminoglycan (GAG) chains, a 4-cysteine-containing region and disulfide bonds near the C-terminus (see reviews by Iozzo, 1997, 1999; Banos et al., 2008; Schaefer and Iozzo, 2008; Merline et al., 2009; Kalamajski and Oldberg, 2010). Of note, the family includes the chondroitin/dermatan sulphate PGs decorin and biglycan (class I SLRPs) and the keratan sulphate containing PGs fibromodulin, keratocan and lumican (class II SLRPs). These molecules have been shown to act as molecular spacers/cross-linkers between collagen fibrils and to protect against cleavage by collagenases thus playing a pivotal role in collagen fibrillogenesis (Geng et al., 2006; Kalamajski and Oldberg, 2007, 2010). Additionally, they serve as matrix reservoirs for soluble signalling molecules by binding a diverse range of bioactive cytokines, morphogens and growth factors, including TGF- $\beta$ (Hildebrand et al., 1994; Merline et al., 2009). They are thus central to the development, growth and function of many highly organised connective tissues including cornea, tendon and skin (Danielson et al., 1997; Svensson et al., 1999; Ameye et al., 2002; Ameye and Young, 2002; Chakravati, 2002; Young et al., 2002; Kao and Liu, 2003; Liu et al., 2003; Reed and Iozzo, 2003; Gealy et al., 2007; Rees et al., 2009; Kalamajski and Oldberg, 2010). Despite the fundamental importance of the SLRPs in regulating collagen fibrillogenesis and tissue growth, surprisingly few studies have focussed upon their involvement in the establishment of the collagenous architecture of the annulus fibrosus. In this study, we set out to (i) describe events in the initial establishment of the collagen framework of the rat annulus at light and electron microscopic levels and (ii) examine the involvement of class I and II SLRPs in ECM assembly/organisational events within the developing annulus.

\section{Materials and Methods}

\section{Source of discal material}

White Wistar rats were killed humanely using a Schedule 1 (Animals Scientific Procedures Act, 1986). Discal material was obtained from foetal (embryonic day [E]15, 16, 17, $18,19,20,21)$, neonate and adult (4 months old) animals (one sample per developmental stage). Lumbar (L)1-L2 IVDs were dissected out of adult spines, whereas foetal and neonatal spines were processed in situ (see below). To facilitate direct comparison between ages and to allow comparative analysis of labelling patterns, consecutive serial sections of the same L1-L2 IVD were used whenever possible.

\section{Histology}

Tissues were fixed in $10 \%$ neutral buffered formol saline, decalcified (from E19 onwards) in $2 \%$ nitric acid until radiologically clear, and then processed into paraffin wax using standard histological methods. Serial sections were cut through the vertebral column/IVD in the sagittal plane and $6 \mu \mathrm{m}$ sections collected onto Histobond glass histology slides (R.A. Lamb, UK/ThermoFisher, www. thermofisher.com). Tissue sections were de-waxed and rehydrated prior to all staining and immunohistochemical labelling procedures.

\section{Picrosirius red staining}

For an appraisal of collagen fibre organisation at the light microscopic level, de-waxed sections were stained in 0.1 $\%(w / v)$ Sirius red F3B in saturated aqueous picric acid. Sections were washed, dehydrated and mounted under coverslips with DPX mountant. Collagen birefringence was then imaged using polarising optics on a Leica DM6000 microscope (Leica Microsystems, Heidelberg, Germany) equipped with a Jenoptik ProgRes C5 colour digital camera (Jenoptik, Jena, Germany). Identical regions of IVD were also photographed in greyscale under Nomarski differential interference contrast optics. The complementary images were then overlaid in Adobe Photoshop allowing an appraisal of both collagen fibre organisation and cellular morphology within the histologically distinct regions of developing IVD. Collagen fibre tilt in the outer annulus was analysed towards the centre of the collagenous lamellae $(n=10)$ and also at their margins, where they attached to the vertebral bodies, using the angle measurement tool in Image $\mathrm{J}$ (NIH, Bethesda, MD, USA). The mean angle of fibre tilt (+/- standard deviation) was then calculated for each region and presented upon line trace diagrams of IVD sections, produced in Microsoft PowerPoint.

\section{Immunohistochemistry}

Dewaxed sections were labelled with the Vector ABC universal immunoperoxidase labelling kit (Vector Laboratories, Burlingame, CA, USA) using a panel of 
Table 1. Antibodies used for immunohistochemistry.

\begin{tabular}{|c|c|c|c|c|}
\hline $\begin{array}{l}\text { Antibody } \\
\text { (dilution) }\end{array}$ & $\begin{array}{l}\text { Clone } \\
\text { (isotype) }\end{array}$ & Pretreatment & Specificity & Source/Reference \\
\hline PR8A4 (1:20) & M (IgG) & $\mathrm{ABC} / \mathrm{K}$ & Biglycan (core protein) & Roughley et al. (1993) \\
\hline $28.4(1: 20)$ & M (IgG1) & $\mathrm{ABC} / \mathrm{K}$ & Decorin (core protein) & Bidanset et al. (1992) \\
\hline KER-1 (1:20) & $\mathrm{M}(\operatorname{IgM\kappa })$ & $\mathrm{ABC} / \mathrm{K}$ & Keratocan (core protein) & $\begin{array}{l}\text { Rees et al. (2009); Gealy et al. (2007); } \\
\text { Kotwal et al. (2007) }\end{array}$ \\
\hline LUM-1 (1:20) & M (IgM) & $\mathrm{ABC} / \mathrm{K}$ & Lumican (core protein) & Kotwal et al. (2007) \\
\hline FIB $(1: 20)$ & $\mathrm{P}$ & $\mathrm{ABC} / \mathrm{K}$ & $\begin{array}{l}\text { Fibromodulin (nine amino } \\
\text { acid peptide sequence at } \\
\text { C-terminus of core protein) }\end{array}$ & Roughley et al. (1996) \\
\hline BKS1 (1:20) & $\mathrm{M}(\operatorname{Ig} M)$ & $\mathrm{ABC} / \mathrm{K}$ & Keratan sulphate "stub" & $\begin{array}{l}\text { Akhtar et al. (2008); Kotwal et al. } \\
\text { (2007) }\end{array}$ \\
\hline
\end{tabular}

ABC, Chondroitinase ABC; K, Keratanase; M, Monoclonal; P, Polyclonal

monoclonal antibodies (mAbs) towards members of the small leucine rich family of PGs (refer to Table 1). Rehydrated tissue sections were circumscribed with a Dako delimiting pen (Dako, Glostrup, Denmark) and immersed in $0.3 \%$ hydrogen peroxide in water $(\mathrm{v} / \mathrm{v})$ for $1 \mathrm{~h}$ to block endogenous peroxidase activity. After washing, sections were treated with normal horse-serum for $30 \mathrm{~min}$ to prevent non-specific antibody binding. Each of the mAbs were then applied to the tissue sections and incubated overnight at $4{ }^{\circ} \mathrm{C}$. Controls were incubated with naive immunoglobulins or the primary antibody was omitted and replaced with phosphate buffered saline (PBS), pH 7.4. All immunolabelling controls were negative showing no non-specific antibody labelling. After overnight incubation in primary antibody, sections were washed in PBS and incubated with biotinylated secondary antibody for $30 \mathrm{~min}$ at room temperature. After washing, sections were then incubated with the avidin, biotin complex for $30 \mathrm{~min}$. Following another wash, NovaRed peroxidase substrate (Vector Laboratories) was added to the sections until the desired colour intensity was developed. Finally, sections were washed, counterstained with haematoxylin and mounted under coverslips with DPX mountant. The resultant immunostaining patterns were photographed under brightfield optics using the Leica microscope described above.

\section{Electron Microscopy}

For an appraisal of collagen organisation at the ultrastructural level, lumbar segments from E15 and E16 foetuses (i.e., developmental stages before and immediately after IVD differentiation, respectively); E19; newborn; and 4 months (adult) IVDs were examined by both transmission and scanning electron microscopy. Samples were fixed initially in $2 \%$ glutaraldehyde and $2 \%$ paraformaldehyde in 0.1 $\mathrm{M}$ cacodylate buffer ( $\mathrm{pH} 7.4)$ for $2 \mathrm{~h}$ at $2{ }^{\circ} \mathrm{C}$.

\section{Transmission electron microscopy}

Fixed samples were washed in buffer and post-fixed in $1 \%$ osmium containing $1.5 \%$ potassium ferrocyanide

Fig. 1. (Next page) Transmitted light microscopy showing the establishment of the collagenous architecture of the annulus fibrosus of the IVD. A-F. Foetal IVD development. Collagen birefringence (orange), visible under polarising optics, has been superimposed upon a Nomarski differential interference contrast micrograph (greyscale) allowing appraisal of collagen fibre organisation and cell morphology respectively. A. Mid-sagittal section of an E15 spine. Birefringence occurs within the fibrillar sheath (arrowhead) that surrounds the notochord (n) and weakly within the cartilage of the vertebral bodies (vb), but not within the IVD condensations (iv). Asterisk denotes pockets of fibrillar matrix within the notochord. B,C. Mid-sagittal section of an E17 IVD; figure C shows detail of boxed area in B rotated through $90^{\circ}$. Collagen birefringence is strongly associated with the oriented laminae of the outer annulus (oa) and with the inferior and superior facets of the nucleus pulposus (np) where they meet the cartilage of the vertebral bodies $(\mathrm{vb})$ in the region of the future vertebral end-plates (ep). D. Sagittal section through the inner annulus at E17. Note parallel bundles of collagen oriented perpendicular to the (vertical) spinal axis, and flanking the nucleus pulposus (outside the plane of section here). E. Sagittal section through the outer annulus at E17. The alternating orientation of collagen fibre bundles associated with successive laminae of the outer annulus manifests itself as a striking crisscross pattern of birefringence. F. Mid-sagittal section through an E19 IVD. Collagen fibre bundles now surround and enclose the nucleus pulposus (np). G-H. Mid and para-sagittal sections, respectively, through a 4 month IVD. N.B. the lamellar organisation of collagen within the disc at this stage reflects that established during foetal development. ep, end-plate; ia, inner annulus; oa, outer annulus; np, nucleus pulposus; vb, vertebral body. Scale bar in microns. 

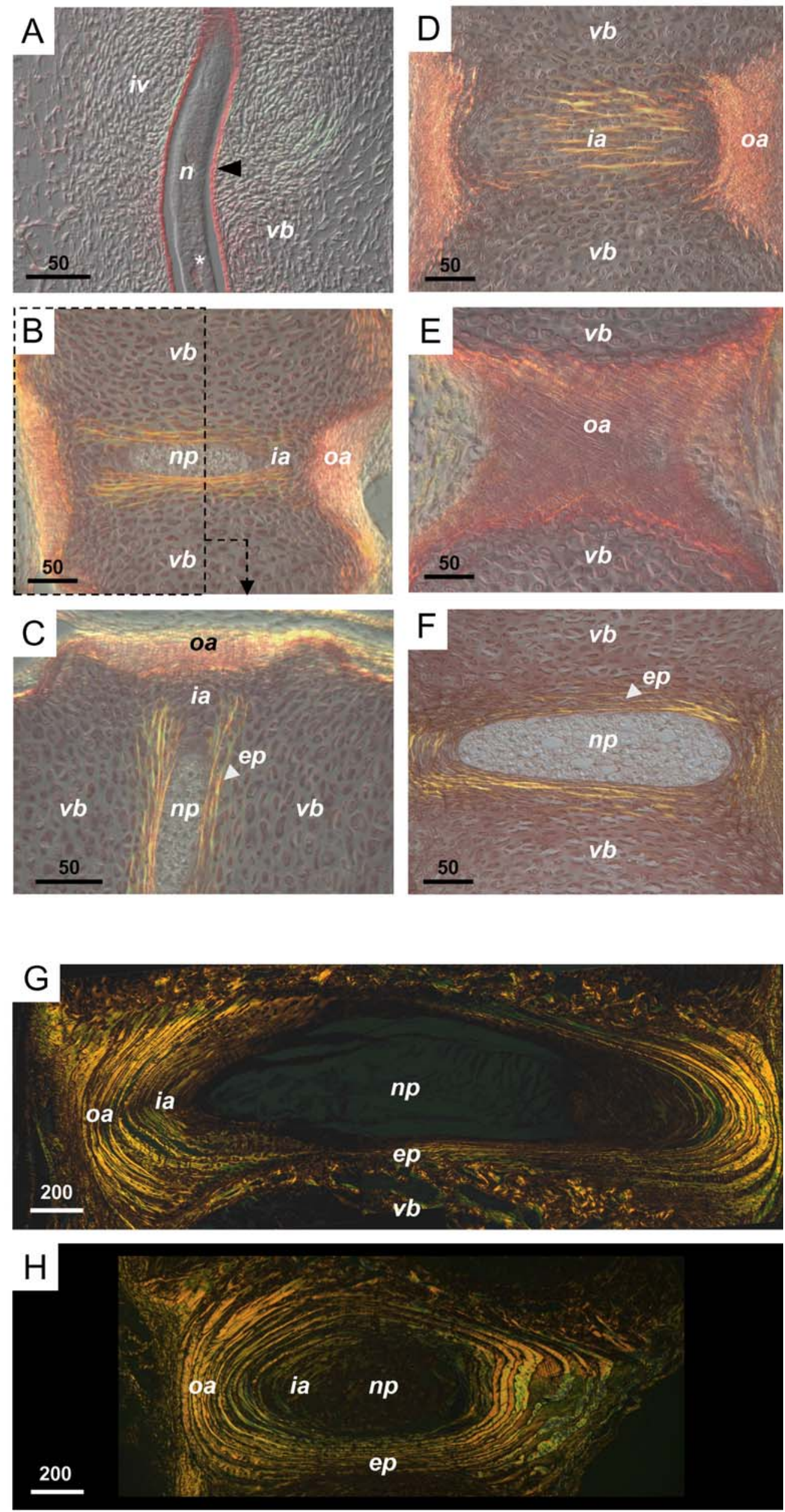
(Sigma-Aldrich, St. Louis, MO, USA) for $1 \mathrm{~h}$ at room temperature. After washing, samples were stained en bloc with $2 \%$ uranyl acetate $(\mathrm{w} / \mathrm{v})$, dehydrated in ethanol, cleared in propylene oxide and infiltrated and embedded in Araldite resin. Ultrathin $60 \mathrm{~nm}$ sections were cut and then stained with $2 \%$ uranyl acetate $(w / v)$ and lead citrate before observation in a Phillips 208 transmission electron microscope (Phillips, Eindhoven, The Netherlands).

\section{Scanning electron microscopy}

Fixed samples were washed in buffer, dehydrated in ethanol and then dried in a critical point dryer before sputter coating in gold using standard electron microscopic procedures. Observation of the coated specimens was in a Phillips XL-20 scanning electron microscope.

\section{Results}

\section{Polarising microscopy}

At embryonic day (E)15, IVDs consisted of segmental condensations of peri-notochordal mesenchyme, separated by the condensing cartilage of the early vertebral bodies. Polarising microscopy of picrosirius red stained sections at this stage showed strong collagen birefringence within the sheath surrounding the notochord (Fig. 1A), with weaker birefringence of the vertebral bodies, but with no evidence of any organised collagen within the intervertebral mesenchyme. By E17 (Fig. 1B,C,D), the notochord had bulged outwards into the intervertebral cell condensations, forming the nucleus pulposus, and the surrounding mesenchyme has differentiated into the histologically-distinct cartilaginous inner and fibroblastic outer parts of the annulus fibrosus. Observation of sections taken at different depths within the foetal IVD revealed striking differences in collagen organisation in the composite disc tissues (Figs. 1,B,C,D). In sections cut close to the mid-sagittal plane (Figs. 1B,C) parallel bundles of birefringent collagen, could be seen juxtaposed between the superior and inferior facets of the nucleus pulposus and vertebral bodies, i.e. in the region of the future vertebral end-plates. These collagen bundles were associated with discoidal-shaped chondrocytes and followed the curvature of the nucleus pulposus, terminating abruptly within the cartilaginous inner annulus. Collagen birefringence was also associated prominently with the oriented fibroblastic laminae of the outer annulus and extended rostro-caudally from the outermost annular laminae to the perichondrium of the vertebral bodies (Figs. 1B,C,D). Sagittal sections through the inner annulus showed the nucleus to be enclosed at its lateral margins by a parallel arrangement of collagen fibre bundles, perpendicular to the spinal axis, that were separated by oval-shaped chondrocytes (Fig. 1D). Sagittal sections though the outer annulus, meanwhile, highlighted the cross-ply organisation of the tissue that was manifest as a striking lattice-like pattern of collagen birefringence (Fig. 1E). Measurement of the angles of collagen bundle fibre tilt showed that towards the centre of the laminae, collagen fibres were oriented at $53.68+/-4.5^{\circ}$ to the spinal axis; increasing to $66.08+/-2.25^{\circ}$ where they inserted into the vertebral bodies (refer to summary Fig. 6). By E19, the annulus had a more lamellar appearance and collagen birefringence was evident between the arching rows of discoidal chondrocytes of the inner annulus (Fig. $1 \mathrm{~F})$. Thus by this stage the nucleus pulposus appeared completely enclosed by collagen fibre bundles and the

Fig. 2. (Next page) Ultrastructural organisation of collagen within the developing annulus. A. Scanning electron micrograph of the superior surface of an E15 spine. Condensations of intervertebral mesenchyme (iv) surround the notochord (n). Cells within the condensation are highly interconnected creating a series of extracellular compartments between cells. B,C. Transmission electron micrographs through the intervertebral cell condensations at E15. B. Cells forming the outer part of the condensation (o) are more flattened in morphology than those towards the inner aspect (i). C. Cells are highly interconnected via stubby cellular extensions (arrowheads) creating a series of conspicuous extracellular compartments between cells (denoted by dashed boundaries). D, E, F. Transmission electron micrographs through the outer annulus at E16. D. Cells have become highly elongate and organised into oriented laminae. The alternating orientation of cells in contiguous laminae is evident. White asterisks denote cells cut transversely within a cell sheet; black asterisks denote those cut longitudinally. E. High power showing the deposition of fibrillar matrix into the extracellular space between contiguous laminae. Large arrow denotes a recess within the plasma membrane into which fibrillar material is deposited. Small arrowhead denotes junction between adjacent cells. m, mitochondrion; nu, nucleus; v, vesicle. F. High power showing alternating orientation of collagen fibrils in the extracellular space between contiguous laminae. Image has been rotated through $90^{\circ}$ with respect to figure D. White asterisks denote collagen fibrils cut transversely; black asterisks denote those cut longitudinally. Dashed lines demarcate the outline of cells in three adjacent laminae. N.B. The orientation of longitudinal collagen fibrils between cells mirrors the orientation of longitudinal stress fibre bundles (sf) within cells. m, mitochondrion; nu, nucleus. G. Section through the nucleus pulposus (np) and inner annulus (ia) of the E19 IVD. Note bundles of fibrillar matrix between arcades of oval shaped chondrocytes within the inner annulus at this stage. H, I. Sections through the inner and outer annulus, respectively, of the IVD at birth. Figure I has been rotated through $90^{\circ}$ with respect to figure H. Collagen fibril bundles between cells have coalesced into rudimentary lamellae. Arrow in $\mathrm{H}$ denotes a cell discharging collagen via a large recess within its cell membrane. White asterisk denotes collagen fibrils bundles cut obliquely; black asterisks denote those cut longitudinally. J,K,L. Scanning electron micrographs showing the superior surface of the annulus at 4 months. ia, inner annulus; oa, outer annulus. J. Low power showing the lamellar organisation of the annulus. K. Higher power showing five distinct collagenous lamellae. White and black asterisks denote alternating fibre orientations in successive lamellae. L. High power of oriented collagen fibre bundles within an individual lamella. Scale bar in microns. 

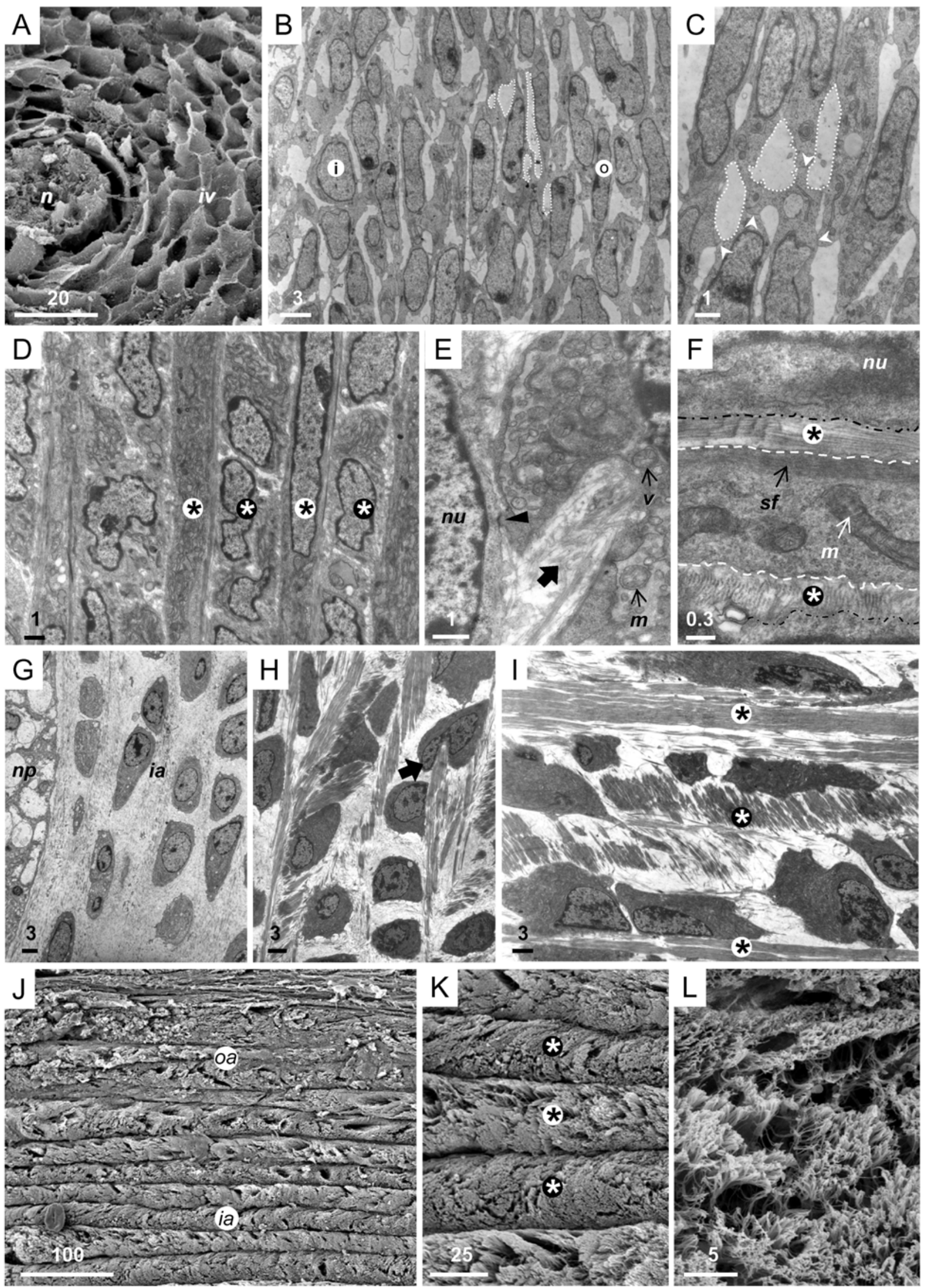
overall organisation of collagen within the distinct discal tissues mirrored that occurring within the adult IVD (Figs. $1 \mathrm{G}, \mathrm{H})$, which has been well-described previously.

\section{Electron microscopy}

Electron microscopic results were supportive of those obtained by polarising/Nomarski DIC light microscopy. At E15, the presumptive IVD consisted of a dense condensation of mesenchymal cells arranged concentrically around the notochord (Fig. 2A,B,C). Cells forming the inner part of the condensation were polygonal in morphology whereas those towards the outside were more flattened and discoidal in shape (Fig. 2B). The cells were intimately associated by many cell-cell junctions throughout the condensation, creating a series of extracellular compartments, but with no evidence of any fibrillar ECM between cells at this stage (Fig. 2A,B,C). At E16, coincident with IVD differentiation, oriented laminae of fibroblasts became identifiable in the outer annulus at the electron microscopic level (Fig. 2D). The laminae consisted of flattened, highly elongate fibroblasts, tightly arranged together and separated by narrow pockets of extracellular space. The orientation of cells in a cell sheet alternated in successive laminae (Fig. 2D) in an identical cross-ply configuration to the pattern of collagen birefringence seen in the outer annulus a day later under polarising optics (Fig. 1E). Higher power observation of the cells in this region showed that they were depositing fibrillar ECM material into the narrow extracellular space between contiguous cell sheets via recesses within their cell membrane (Fig. 2E). Furthermore, the orientation of the deposited fibrillar ECM material matched the orientation of the cells that were secreting it and also their underlying intracellular stress fibres (Fig. 2F). By E19, the lamellar organisation of the inner annulus, seen by polarised/DIC light microscopy (Fig. $1 \mathrm{~F}$ ), was also evident at the ultrastructural level (Fig. $2 \mathrm{G})$. The cells were oval to polygonal in morphology and separated by tracts of fibrillar ECM, representing nascent collagenous lamellae. By birth, these lamellae had become reinforced by progressive accretion of secreted collagen, and the alternating orientation of ECM in contiguous lamellae was obvious in both the inner and outer annulus (Figs. 2H and 2I, respectively). Observation of 4 month IVDs by scanning electron microscopy (Fig. 2J,K,L) revealed a well developed and highly ordered radial-ply organisation of oriented collagenous lamellae that has been described previously. High power observation showed the alternating orientation of collagen fibre bundles in adjacent lamellae (Figs 2K,L), set up initially during early foetal development.

\section{Immunohistochemistry of small leucine rich proteoglycans within the disc}

\section{Foetal disc SLRP Labelling Patterns}

Biglycan and decorin had broadly similar distributions within the IVD during foetal development (Fig. 3). Both SLRPs were weakly detectable within the cartilage of the vertebral bodies and notochordal sheath at E15 (Fig. 3A,F), with decorin, but not biglycan, weakly identifiable within the intervertebral mesenchyme (Fig. 3F). At E17, after IVD differentiation had occurred, both SLRPS were associated with cells of the vertebral bodies and nucleus pulposus (Fig. 3B,G) and by E19 both SLRPs became more visible within the cartilage matrix of the vertebral bodies and inner annulus. At E21, both biglycan (Fig. $3 \mathrm{D}, \mathrm{E})$ and decorin (Fig I,J) became strongly associated with the cartilage matrix of the vertebral bodies and inner annulus with weaker labelling of the outer annulus (Fig. $3 \mathrm{E}, \mathrm{J})$. Biglycan, in particular, was more prominent within the interface zone between these tissues (Fig, 3E).

Keratocan was not detected within the IVD at any stages during foetal development (Fig. 4 A-E).

Lumican was not detectable above background levels at E15 (Fig. 4F), but appeared weakly associated with cells of the vertebral bodies and nucleus pulposus at E17 (Fig. $4 \mathrm{G})$. Lumican became more visible within the cartilage matrix of the vertebral bodies and inner annulus during the remainder of foetal development (Fig. 4H -J), and by E21 was also weakly identifiable within the outer annulus (Fig. 4J).

Fibromodulin was the most prominent of the SLRPs detected within the developing IVD, particularly within the annulus (Fig. 4K-O). At E15, it was not detectable within the intervertebral cell condensations, vertebral body or notochord (Fig. 4K). By E17, following IVD differentiation, it occurred prominently throughout the cartilage matrix of the inner annulus, circumscribing the nucleus, and extending into the insertional region where annulus interfaced with vertebral bodies. Apart from at their superior and inferior surfaces, the vertebral bodies were negative, as was the nucleus pulposus (Fig. 4L). Weaker fibromodulin labelling was also evident in the fibroblastic outer annulus at this stage, suggestive of the involvement of this SLRP in the initial organisation of oriented collagenous lamellae (Fig. 4L). By E19, the extent of fibromodulin labelling extended rostro-caudally into the cartilage matrix of the vertebral bodies, but the distribution remained unchanged within the IVD (Fig. 4M). At E21, prominent fibromodulin label occurred throughout the annulus fibrosus, particularly where the developing annular lamellae interfaced with the cartilage of the vertebral bodies.

Comparison of the SLRP labelling patterns with those of keratan sulphate showed that KS occurred exclusively within the inner annulus during foetal IVD development (Fig. 4P-T). Thus, it had a considerably more restricted distribution with respect to the SLRP core proteins to which it has known association (i.e., keratocan, lumican and fibromodulin).

\section{Adult disc SLRP labelling patterns}

At 4 months, the SLRPs, had remarkably similar and broad distributions within the IVD (Figs. 5A-D and 5G-J); with the notable exception of keratocan, which was absent from the disc but was highly prominent in the adjacent spinal tissue (Fig. 5E,F). Biglycan (Figs. 5A,B), decorin (Figs. 5C,D), lumican (Figs. 5G,H) and fibromodulin (Figs. $5 \mathrm{I}, \mathrm{J})$ had widespread distributions within the ECM of the annulus fibrosus, nucleus pulposus and vertebral growth plate; however, there were subtleties in their patterns of expression and also their intensity of label. For instance, 


\section{Biglycan}

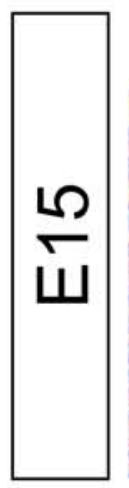

프

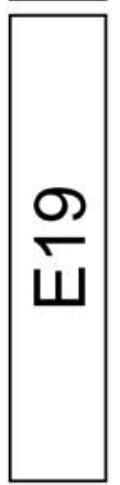

\begin{tabular}{|l}
\hline \\
U \\
\end{tabular}
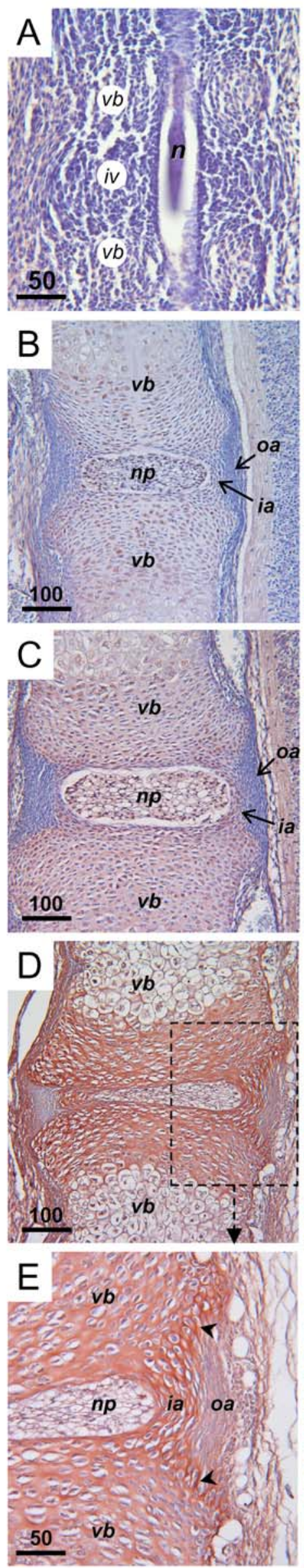

\section{Decorin}
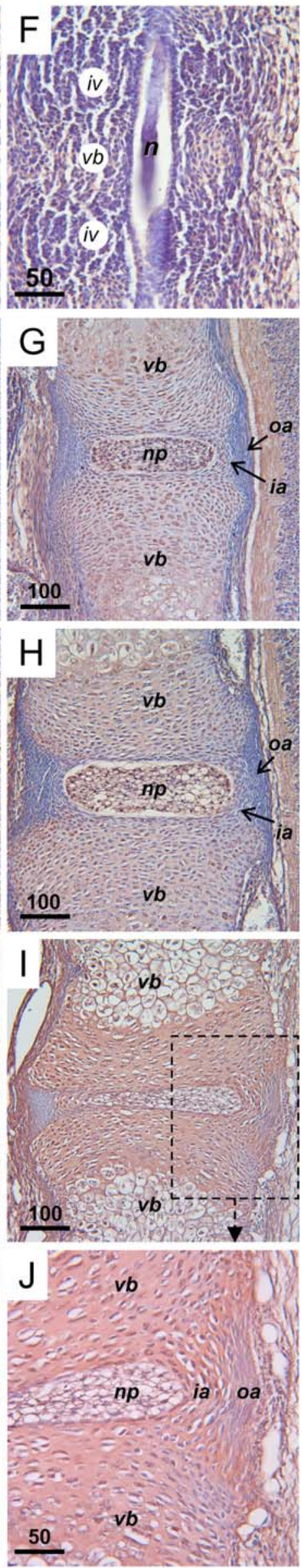

Fig. 3. Immunohistochemical distributions of Class I SLRPs during foetal IVD development. Immunoperoxidase $\&$ haematoxylin. Boxed areas in figs $\mathbf{D}$ $\& \mathbf{I}$ are presented in detail in subjacent panel. A-E. Biglycan (mAb PR8A4). F-J. Decorin (mAb 28.4). Both SLRPs have similar distributions within the IVD and vertebral bodies during foetal development (E15-E21), occurring primarily with the developing cartilages of the vertebral bodies and inner annulus. Biglycan and decorin are both weakly expressed in the outer annulus at the E21 stage, with stronger labelling of biglycan, in particular, at the interface between annulus fibrosus and vertebral bodies (arrow head). iv, intervertebral mesenchyme; ia, inner annulus; $\mathrm{n}$, notochord; np, nucleus pulposus; vb, vertebral body. Scale bar in microns. 
Keratocan
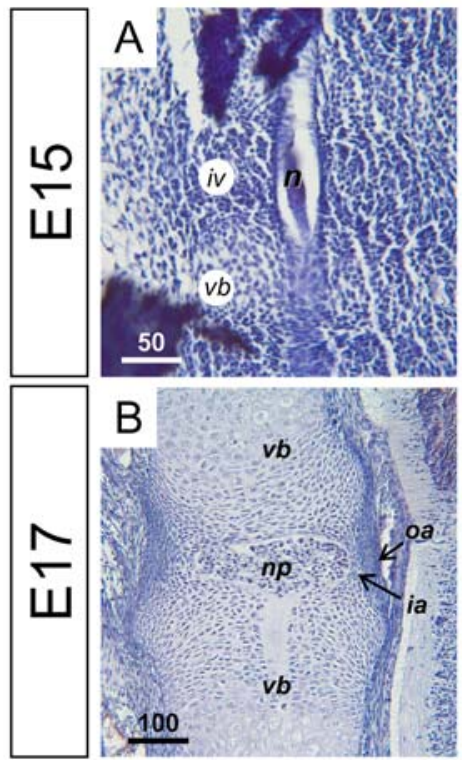

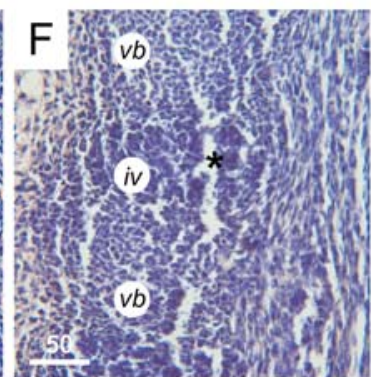

Fibromodulin

KS
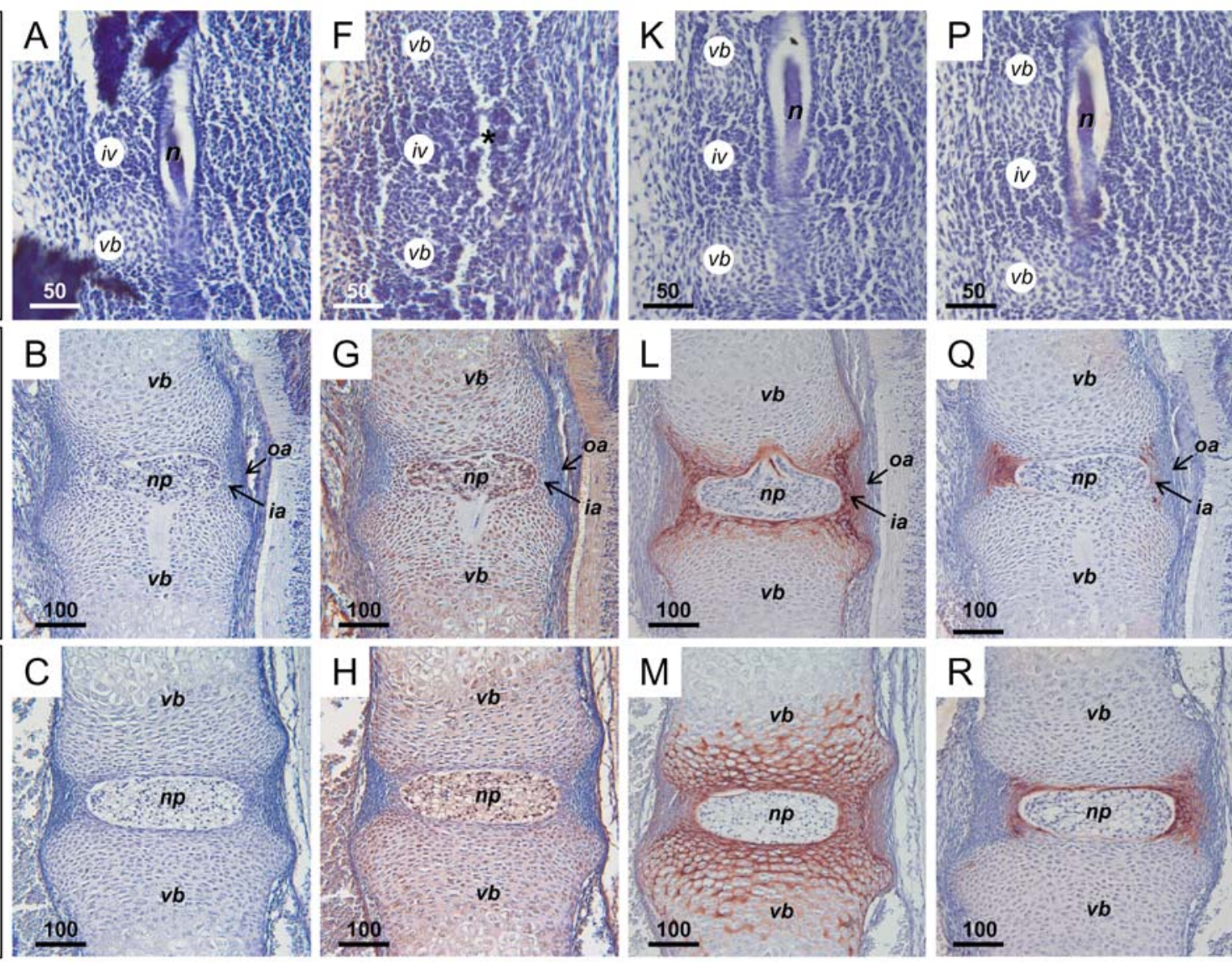

$\mathrm{R}$
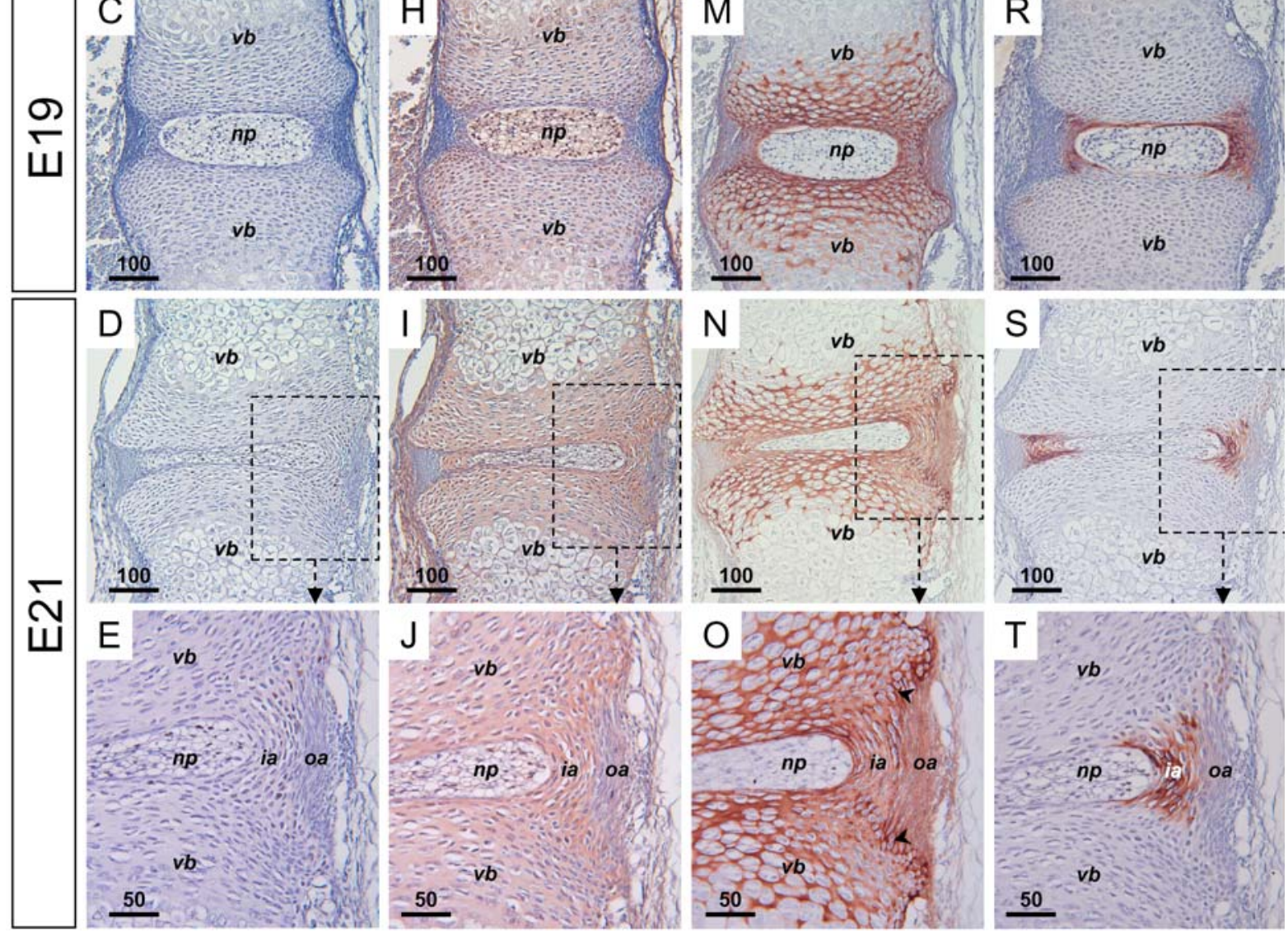

Fig. 4. Immunohistochemical distributions of Class II SLRPs and keratan sulphate during foetal IVD development. Immunoperoxidase \& haematoxylin. Boxed areas in figs $\mathbf{D}, \mathbf{I}, \mathbf{N} \& \mathbf{S}$ are presented in detail in subjacent panel. A-E. Keratocan (mAb KER-1). Keratocan (A-E) is not detected within the IVD or vertebral bodies during foetal development. F-J. Lumican (mAb LUM-1). Lumican is weakly detectable in the cartilages of the vertebral bodies and inner annulus, and also the nucleus pulposus. By E21 it is also weakly expressed within the outer annulus. K-O. Fibromodulin (mAb anti-FIB). Fibromodulin occurs prominently within the annulus during foetal development, but is absent from the nucleus pulposus. At E17 it is present within the inner annulus and at the interface between IVD and vertebral bodies, circumventing the nucleus, with weaker label extending into the outer annulus. By E19, label extends rostro-caudally into the cartilaginous ends of the vertebral bodies and at E21 it is prominent in the outer annulus, particularly at its attachment to the vertebral bodies (arrowhead) P-T. Keratan sulphate (mAb BKS-1). Keratan sulphate occurs very specifically within the cartilage of the inner annulus during foetal development, thus has a much more restricted distribution compared to the class II SLRPs examined. iv, intervertebral mesenchyme; ia, inner annulus; n, notochord; np, nucleus pulposus; vb, vertebral body. Scale bar in microns. 

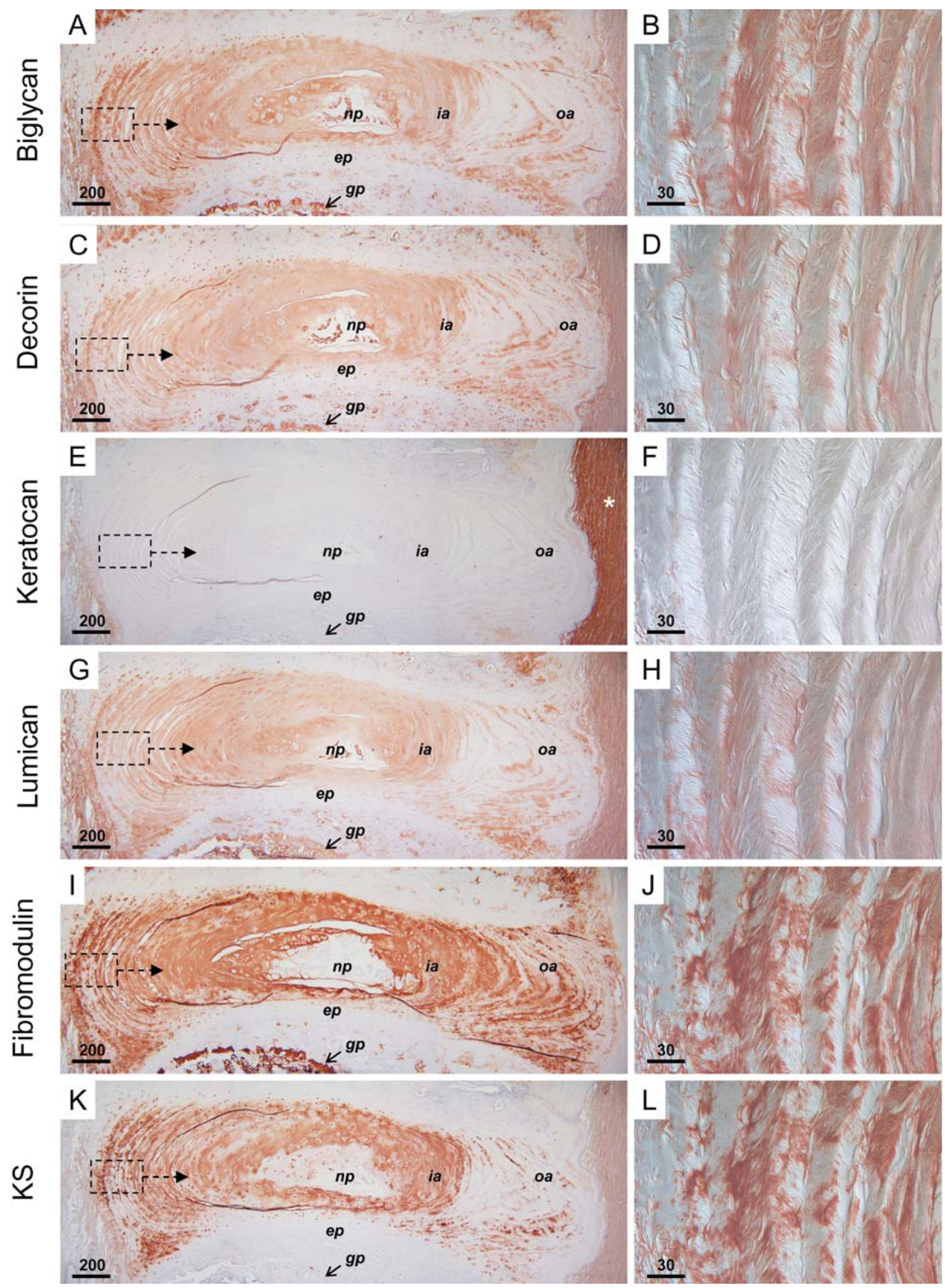

Fig. 5. Immunohistochemical distributions of Class I and II SLRPs and keratan sulphate in the 4 month rat IVD. Immunoperoxidase labelling. A, B. Biglycan (mAb PR8A4). C, D. Decorin (mAb 28.4) E, F. Keratocan (mAb KER1). G, H. Lumican (mAb LUM-1). I, J. Fibromodulin (mAb anti-FIB). K, L. Keratan sulphate (mAb BKS-1). Boxed areas of outer annulus depicted in left panel are shown in detail in right panel. With the exception of keratocan, which is absent from the IVD but present in the adjacent spinal cord (white asterisk), all SLRPS examined have similar widespread distributions within the IVD, cartilage endplate and vertebral growth plate after 4 months (left panel). In the annulus, they are intimately associated with constituent collagen fibre bundles of contiguous lamellae (right panel). Note absence of fibromodulin label in notochordal tissue of nucleus pulposus (np). Also note lack of KS label associated with cartilage end-plate (ep) and vertebral growth plate (gp). ia, inner annulus; oa, outer annulus. Scale bar in microns. 


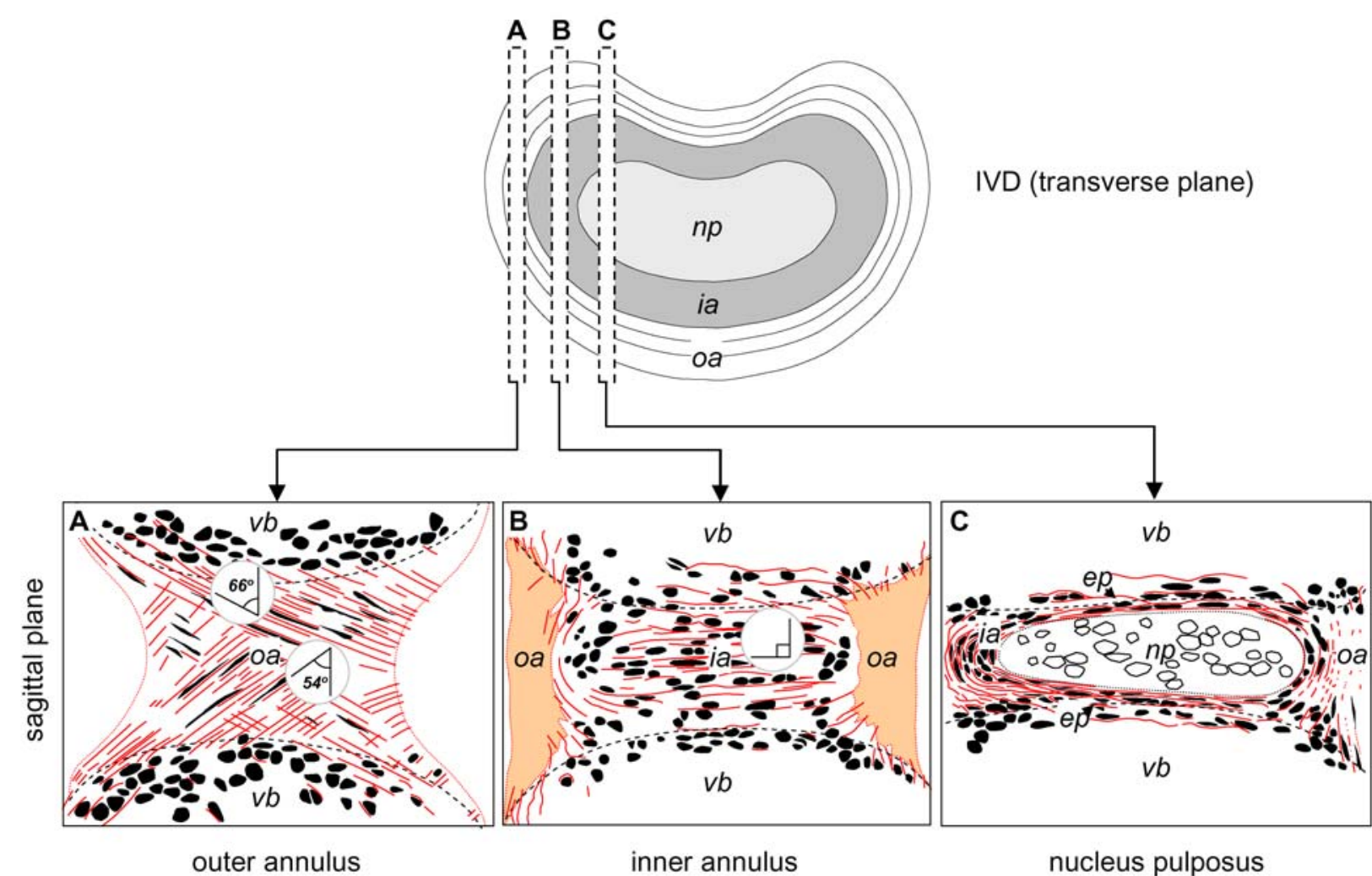

Fig. 6. Schematic summarising the early collagenous organisation of the foetal lumbar IVD. Top graphic. Idealised lumbar IVD in transverse plane illustrating tissue zones and section planes $(\mathbf{A}, \mathbf{B}, \mathbf{C})$ depicted in the underlying schematics. A, B, C. Schematics illustrating collagen orientation (red lines) and cell morphology (black fills) within the different discal/vertebral tissues from the section planes depicted in the above graphic. Black dashed lines represent the interface between IVD and vertebral bodies. Angles of collagen fibre tilt associated with different regions of outer and inner annulus are shown inset. A. Sagittal section through the outer annulus (oa) reveals the alternating orientation of collagen fibres associated with successive cell laminae at E17. These fibres insert into the cartilaginous vertebral bodies (vb) B. Sagittal section through the inner annulus (ia) at E17 reveals that the nucleus pulposus (outside plane of section here) is flanked by a parallel arrangement of collagen fibre bundles that run perpendicular to the spinal axis. C. Sagittal section through the nucleus pulposus (np) of the disc at E19. Parallel bundles of collagen within the presumptive vertebral end-plates merge with those of the inner annulus (ia). Thus the nucleus pulposus becomes enclosed by a collagen 'cage'.

biglycan and decorin were also associated with cells of the cartilage end-plate (Figs 5A,C, respectively) and fibromodulin, which was highly prominent throughout the annulus, was noticeably absent from the notochordal nucleus pulposus (Fig. 5I). High power observation showed that biglycan (Fig. 5B), decorin (Fig. 5D), lumican (Fig. 5H) and fibromodulin (Fig. 5J) also had similar distributions within the annulus at this stage, all being intimately associated with the collagen fibre bundles of annular lamellae. Comparison of the SLRP labelling patterns at 4 months (Figs. 5A-J) with those of KS (Figs. $5 \mathrm{~K}, \mathrm{~L})$ again showed the latter to have a more restricted tissue distribution, although less so than in foetal stages. KS was present very specifically within the discal tissues and was absent from the cartilage end-plate, vertebral growth plate and vertebral bodies.

\section{Discussion}

The organisation of collagen in the annulus fibrosus of the mature IVD is well documented (Inoue and Takeda, 1975;
Hashizume, 1980; Inoue, 1981; Hickey and Hukins, 1980; Hukins and Meakin, 2000). To our knowledge this is the first study that has examined, at both light and electron microscopic levels, (i) how the organisation of collagen within the distinct discal tissues becomes established during foetal development, and (ii) the roles that class I and II SLRPs may play in this process.

Previous studies have shown that the organisation of oriented collagen in the outer annulus is preceded by an identical arrangement of oriented cells that are precisely arranged into laminae (Peacock et al., 1951a; Walmsley 1953; Rufai et al., 1995; Hayes et al., 1999). Underlying this cellular organisation is a subcellular cytoskeletal template: an integrated network of oriented intracellular actin stress-fibres linked intercellularly via adherens junctions (Hayes et al., 1999). These appear to direct the initial cell orientation process in the outer annulus and may be involved subsequently in the organised deposition of ECM (see below). The present study shows that a period of rapid, highly organised matrix synthesis and deposition follows the initial cellular orientation phase. By birth, the organisation of collagen is well established and the lamellar 
structures then appear to expand by progressive, ordered matrix accretion.

The initial cellular orientation phase within the outer annulus is clearly a pivotal moment in disc development and an essential prerequisite for the subsequent lamellar organisation of oriented collagen. In the E15 rat, before notochordal bulging, there is no ultrastructurally detectable fibrillar collagen within the cellular disc condensations, confirming previous immunofluorescence studies (Rufai et al., 1995; Hayes et al., 1999, 2001). After notochordal bulging, there is rapid up-regulation of collagen deposition: type II within the inner annulus and type I in the outer (Rufai et al., 1995; Hayes et al., 2001). The ultrastructural and polarising studies undertaken here augment our previous investigations and demonstrate fibrillar collagen deposition between cell sheets of the outer annulus immediately following the initial cellular orientation phase, with an extensive well-organised collagen fibre architecture emerging within the annulus by E17. The coincident expression of fibromodulin at E17 during the subsequent matrix assembly and organisation process within the annulus suggests that this SLRP, in particular, may be involved in modulating aspects of collagen fibrillogenesis and foetal growth of annular lamellae (see below).

The angle of collagen fibre tilt within the oriented lamellae of the outer annulus is highly significant to adult annulus function and has important ramifications for repair/regeneration of the tissue following disease/injury (Hukins and Meakin, 2000; Nerurkar et al., 2010; Smith et al., 2011): the collagen fibres have to form a pressure vessel to retain the nucleus, and to resist compression, torsion and flexion. Towards the midline of foetal discs we observe an angle of $54^{\circ}$ with respect to the long axis of the spine, conforming closely to the theoretical 'magic angle' required to achieve optimal isotensoidal loading of fibre-reinforced cyclindrical laminates $\left(54.7^{\circ}\right.$; Hukins and Meakin, 2000). This angle increases to $66^{\circ}$ where the annular lamellae intercalate with the vertebral bodies; an angle that correlates very closely with that measured by X-ray diffraction of foetal human lumbar discs $\left(65^{\circ}\right.$; Hickey and Hukins, 1980). The discrepancy between these angles probably relates to the elliptical path that the fibres take around the annulus and has been noted previously (Marchand and Ahmed, 1990). Of relevance, it has been shown that the $65^{\circ}$ angle of fibre tilt measured by x-ray diffraction within the human annulus remains constant in the same anatomical disc region from foetus to adult (Hickey and Hukins, 1980). If we accept that the angle of collagen fibre tilt within the annulus is determined by the orientation of fibroblasts at the early stages of disc differentiation, then it prompts the question of just how early in development is the angle determined? Is the angle established during notochordal bulging as a cellular response (e.g., the formation of oriented stress fibres) to mechanical loading as the nucleus expands? Or is it established even earlier, perhaps by the pattern of cellcell interactions in the early annulus prior to notochordal expansion, which might suggest the involvement of the molecular genetic mechanisms associated with spinal patterning?
The cellular mechanisms that underlie the organised deposition of matrix within the outer annulus appear similar, at least superficially, to those occurring in other oriented connective tissues (reviewed by Banos et al., 2008). In tendon and cornea, collagen fibrils self-assemble within recesses of the plasma membrane; their lateral accretion into fibres, bundles and fascicles/lamellae then occurs via a series of extracellular compartments formed between cells (Birk and Trelstad, 1984, 1986). Canty and colleagues (Canty and Kadler, 2002; Canty et al., 2004) described a cellular structure in early developing tendons involved in the very earliest stages of oriented collagen secretion, the fibripositor. We have not observed fibripositors, although the methodology necessary to see them involves extensive section series, thus the chance of observing them in our study would be slim. However, we do show a high degree of cell-to-cell contact within the IVD condensations at E15, resulting in the formation of extensive membrane-bound intercellular space that is initially devoid of any obvious fibrillar ECM. This membrane-bound space appears to be reorganised during the cellular orientation phase at E16, and may provide an intercellular template into which the oriented fibroblasts then secrete and organise their collagen. Collagen may be shuttled in vesicles to the cell surface via oriented actin fibres (DePina and Langford, 1999; Gealy et al., 2009) and deposited into the compartmentalised space between contiguous laminae; self assembling into oriented fibrils at the cell surface (Birk and Trelstad, 1984; 1986; Canty and Kadler, 2002; Canty et al., 2004; Banos et al., 2008). This is supported by our ultrastructural data: collagen fibrils appear to be secreted into the narrow space between contiguous laminae via in-pushing of the plasma membrane; the orientation of the cells - and their underlying stress fibres - matching the orientation of the collagen that is secreted.

The results also show that both the inner annulus and presumptive endplates have a high degree of matrix orientation. The initial parallel bundles of collagen within the endplates are later connected by collagen of the inner annulus. This, along with the collagen bundles seen around the periphery of the nucleus pulposus indicates the formation of a collagen "cage" enclosing the nucleus pulposus. This becomes strengthened considerably during development, forming a distinct capsule around the nucleus (Hashizume, 1980; Inoue and Takeda, 1981). This reinforcing structure may derive, initially, from the collagenous sheath that surrounds the notochord. As the notochord expands outwards it causes reorganisation of the surrounding tissue and triggers expression of GAGs and large PGs by cells forming the inner annulus (Rufai et al., 1995; Hayes et al., 2001). Further mechanical signals are likely to derive from onset of muscle activity in the spine (Panattoni and Todros, 1989), leading to compressive loading of the newly formed IVDs and consequent reinforcement of the enclosing structures.

The SLRPs, with the exception of fibromodulin, were weakly represented within the developing cartilage of the vertebral column, becoming generally more prominent in the adult. They are known to be key regulators of cartilage 
differentiation, via matrix sequestration of growth factors and through control of collagen fibrillogenesis (Banos et al., 2008; Kalamajski and Oldberg, 2010). Thus, it is likely that they are involved in growth and differentiation of the cartilages of the vertebral body, endplate and inner annulus during early spine development.

All of the class I and II SLRPs have known affinities with fibrillar collagen and are important in growth factor binding in a variety of oriented connective tissues including, cornea, tendon and skin (Danielson et al., 1997; Svensson et al., 1999; Ameye et al., 2002; Ameye and Young, 2002; Chakravarti, 2002; Young et al., 2002; Kao and Liu, 2003; Rees et al., 2009). In cornea, where the collagen fibre diameter and spacing must be tightly controlled to ensure transparency, the SLRPs are well represented (Hassell and Birk, 2010; Kao and Liu, 2003). With its purely mechanical role, the outer annulus may not require this level of control over fibrillogenesis; particularly during foetal development, when the lamellae are only starting to become established.

The prominent expression of fibromodulin during foetal disc development has been noted previously in the mouse at E14.5 (Aszodi et al., 1998) and also during growth and aging of human IVDs (Sztrolovics et al., 1999). This study shows that fibromodulin is the only SLRP that is present in the outer annulus from its early differentiation and its expression coincides with the onset of collagen fibrillogenesis and organisation (Hayes et al., 1999). Fibromodulin has established roles in collagen fibrillogenesis and assembly, binding collagen types I, II and XII (Hedbom and Heinegård, 1989; Font et al., 1996, 1998; Kalamajski and Oldberg, 2007; Viola et al., 2007), and thus may be important in collagen assembly in the developing lamellae. The importance of fibromodulin in collagen fibrillogenesis is highlighted in the fibromodulin-null mouse, whose tendons display abnormal collagen fibrils, tissue organisation and altered lumican deposition (Svensson et al., 1999). Surprisingly, the tissue organisation of their IVDs has not yet been studied; however, the results of our study suggest that there may be similar pathological manifestations within the annulus fibrosus of these animals. Fibromodulin is also essential for the binding and sequestration of growth factors within extracellular matrices (Svensson et al., 1999; 2000), including modulation of TGF- $\beta$ (Hildebrand et al., 1994; Zheng et al., 2011); which is known to have a profound effect on the differentiation of outer annulus cells (Hayes and Ralphs, 2011).

A notable feature of this study is the major difference in labelling patterns between fibromodulin and KS distribution during foetal development: fibromodulin is more widely distributed than KS, the latter being restricted specifically to inner annulus. This suggests that the fibromodulin core protein may be substituted with GAG species other than KS (e.g., chondroitin sulphate/dermatan sulphate) during foetal development (Roughley et al., 1996; Sztrolovics et al., 1999; Hayes et al., 2001, 2011). It is unlikely that antigen masking explains the difference, as the KS antibody used here (BKS-1) recognises a keratanase-generated neoepitope 'stub'. Furthermore, the KS labelling patterns we report here are identical to those reported in our previous studies (Rufai et al., 1995; Hayes et al., 2001) that used antibodies recognising distinct epitopes within 'native', intact KS chains (Caterson et al., 1983; Craig et al., 1987). As further support for this, we see a more widespread distribution of KS label in adult discs and a closer correlation with fibromodulin label (as well as other class II SLRPs, excluding keratocan). This suggests greater KS glycosylation of SLRPs during later growth and differentiation of the rat disc. Differences in glycosylation may have effects on specific roles of SLRPs, as we have previously suggested for chondroitin sulphate GAGs (Hayes et al., 2011). The differential distributions of KS and fibromodulin (as well as the other class II SLRPs) within the foetal IVD might therefore reflect functional specialisation of these SLRPs during skeletogenesis.

The expression of SLRPS in the outer annulus becomes more complex with age, with all but keratocan becoming widely expressed. This may be associated with the fact that between birth and adulthood the outer annulus undergoes metaplasia to fibrocartilage (Rufai et al., 1995, Hayes et al., 1999, Melrose et al., 2001). The broad, overlapping distributions of SLRPs within the adult disc, coupled with the potential for substitution, would permit wide interaction with a host of different cytokines, growth factors and many other ECM components, leading to modulation of cellular function. SLRP members may act as matrix reservoirs, regulating the activity of different growth factors such as TGF- $\beta$ and IGF1 that may drive the fibrocartilaginous differentiation of the annulus (Hayes and Ralphs, 2011) and may be involved in the chondrogenic development of the nucleus pulposus, in addition to roles in fibrillogenesis (Melrose et al., 2001). The presence of SLRPs, with the exception of fibromodulin, within the notochordal component of the nucleus pulposus meanwhile underlines the importance of this tissue as a signalling centre within the disc (Aguiar et al., 1999; Erwin and Inman, 2006; Erwin et al., 2006) and highlights the subtley distinct roles that different SLRP family members may play in IVD growth and development.

In summary, this study adds significantly to our previous investigations (Rufai et al., 1995; Hayes et al., 1999, 2001, 2011; Hayes and Ralphs, 2011) that describe developmental processes involved in the establishment and growth of the annulus fibrosus of the IVD. The developmental mechanisms underlying annulus formation and growth have wider relevance to other oriented connective tissues, including cornea and tendon, as well as having significant ramifications for improved strategies for connective tissue repair and regeneration (O'Halloran and Pandit, 2007; Masuda and Lotz, 2010; Nerurkar et al., 2010).

\section{References}

Aguiar DJ, Johnson SL, Oegema TR (1999) Notochordal cells interact with nucleus pulposus cells: regulation of proteoglycan synthesis. Exp Cell Res 246: 129-137.

Akhtar S, Kerr BC, Hayes AJ, Hughes CE, Meek KM, Caterson B (2008) Immunochemical localization of keratan sulphate proteoglycans in cornea, sclera, and limbus using 
a keratanase-generated neoepitope monoclonal antibody. Invest Ophthalmol Vis Sci 49: 2424-2431.

Ameye L, Young MF (2002) Mice deficient in small leucine-rich proteoglycans: novel in vivo models for osteoporosis, osteoarthritis, Ehlers-Danlos syndrome, muscular dystrophy, and corneal diseases. Glycobiol 12: 107-116.

Ameye L, Aria D, Jepsen K, Oldberg A, Xu T, Young MF (2002) Abnormal collagen fibrils in tendons of biglycan/fibromodulin-deficient mice lead to gait impairment, ectopic ossification, and osteoarthritis. FASEB J 16: 673-680.

Aszódi A, Chan D, Hunziker E, Bateman JF, Fässler $\mathrm{R}$ (1998) Collagen II is essential for the removal of the notochord and the formation of intervertebral discs. J Cell Biol 143: 1399-1412.

Banos CC, Thomas AH, Kuo CK (2008) Collagen fibrillogenesis in tendon development: current models and regulation of fibril assembly. Birth Defects Res 84: 228-244.

Bidanset D, Guidry C, Rosenberg L, Choi H (1992) Binding of the proteoglycan decorin to collagen type-VI. J Biol Chem 267: 5250-5256.

Birk DE, Trelstad RL(1984) Extracellular compartments in matrix morphogenesis: collagen fibril, bundle, and lamellar formation by corneal fibroblasts. J Cell Biol 99: 2024-2033.

Birk DE, Trelstad RL(1986) Extracellular compartments in tendon morphogenesis: collagen fibril, bundle, and macroaggregate formation. J Cell Biol 103: 231-240.

Bogduk N (2005) Clinical Anatomy of the Lumbar Spine and Sacrum. 4th ed. Churchill Livingstone, London.

Canty EG, Kadler KE (2002) Collagen fibril biosynthesis in tendon: a review and recent insights. Comp Biochem Physiol 133: 979-985.

Canty EG, Lu Y, Meadows RS, Shaw MK, Holmes DF, Kadler KE (2004) Co-alignment of plasma membrane channels and protrusions (fibripositors) specifies the parallelism of tendon. J Cell Biol 165: 553-563.

Caterson B, Christner JE, Baker JR(1983) Identification of a monoclonal antibody that specifically recognizes corneal and skeletal keratan sulphate. Monoclonal antibodies to cartilage proteoglycan. J Biol Chem 258: 8848-8854.

Chakravarti S (2002) Functions of lumican and fibromodulin: lessons from knockout mice. Glycoconj J 19: $287-293$.

Craig FM, Ralphs JR, Bentley G, Archer CW (1987) MZ15, a monoclonal antibody recognizing keratan sulphate, stains chick tendon. Histochem J 19: 651-657.

Danielson KG, Baribault H, Holmes DF, Graham H, Kadler KE, Iozzo RV (1997) Targeted disruption of decorin leads to abnormal collagen fibril morphology and skin fragility. J Cell Biol 136: 729-743.

DePina AS, Langford GM (1999) Vesicle transport: the role of actin filaments and myosin motors. Microsc Res Tech 47: 93-106.

Erwin WM, Inman RD (2006) Notochordal cells regulate disc chondrocyte proteoglycan production and cell proliferation. Spine 31: 1094-1099.
Erwin WM, Ashman K, O’Donnel P, Inman RD (2006) Nucleus pulposus notochordal cells secret connective tissue growth factor and up-regulate proteoglycan expression by intervertebral disc chondrocytes. Arthritis Rheum 54: 3859-3867.

Eyre DR, Muir H (1977) Quantitative analysis of types I and II collagens in human intervertebral discs at various ages. Biochim Biophys Acta 492: 29-42.

Font B, Eichenberger D, Rosenberg LM, Van der Rest M (1996) Characterization of the interactions of type XII collagen with two small proteoglycans from fetal bovine tendon, decorin and fibromodulin. Matrix Biol 15: 341348.

Font B, Eichenberger D, Goldschmidt D, Boutillon MM, Hulmes DJS (1998) Structural requirements for fibromodulin binding to collagen and the control of type I collagen fibrillogenesis. Critical roles for disulphide bonding and the C-terminal region. Eur J Biochem 254: 580-587.

Gealy EC, Kerr BC, Young RD, Tudor D, Hayes AJ, Hughes CE, Caterson B, Quantock AJ, Ralphs JRR (2007) Differential expression of the keratan sulphate proteoglycan, keratocan, during chick corneal embryogenesis. Histochem Cell Biol 128: 551-555.

Gealy C, Hayes AJ, Buckwell R, Young RD, Caterson B, Quantock AJ, Ralphs JR (2009) Actin and type I collagen propeptide distribution in the developing chick cornea. Invest Ophthalmol Vis Sci 50: 1653-1658.

Geng Y, McQuillan D, Roughley PJ (2006) SLRP interaction can protect collagen fibrils from cleavage by collagenases. Matrix Biol 25: 484-491.

Hashizume H (1980) Three-dimensional architecture and development of lumber intervertebral discs. Acta Med Okayama 34: 301-314.

Hassell JR, Birk DE (2010) The molecular basis of corneal transparency. Exp Eye Res 91: 326-335.

Hayes AJ, Ralphs JR (2011) The response of foetal annulus fibrosus cells to growth factors: modulation of matrix synthesis by TGF- $\beta 1$ and IGF-1. Histochem Cell Biol, in press.

Hayes AJ, Benjamin M, Ralphs JR (1999) Roles of actin stress fibres in the development of the intervertebral disc: Cytoskeletal control of extracellular matrix assembly. Dev Dyn 215: 179-189.

Hayes AJ, Benjamin JR, Ralphs JR (2001) Extracellular matrix in development of the intervertebral disc. Matrix Biol 20: 107-121.

Hayes AJ, Hughes C, Ralphs RR, Caterson B (2011) Chondroitin sulphate sulphation motif expression in the ontogeny of the intervertebral disc. Eur Cells Mater 21: $1-14$.

Hedbom E, Heinegård D (1989) Interaction of a 59$\mathrm{kDa}$ connective tissue matrix protein with collagen I and collagen II. J Biol Chem 264: 6898-6905.

Hedbom E, Heinegård, D (1993) Binding of fibromodulin and decorin to separate sites on fibrillar collagens. J Biol Chem 268: 27307-27312.

Hickey DS, Hukins DWL (1980) X-Ray diffraction studies of the arrangement of collagenous fibres in human fetal intervertebral discs. J Anat 131: 81-90. 
Hildebrand A, Romarís M, Rasmussen LM, Heinegård D, Twardzik DR, Border WA, Ruoslahti E (1994) Interaction of the small interstitial proteoglycans biglycan, decorin and fibromodulin with transforming growth factor $\beta$. Biochem J 302: 527-534.

Hukins DWL, Meakin JD (2000) Relationship between structure and mechanical function of the tissues of the intervertebral joint. Amer Zool 40: 42-52.

Inoue H (1981) Three-dimensional architecture of lumbar intervertebral discs. Spine 6: 139-146.

Inoue H, Takeda T (1975) Three-dimensional observation of collagen framework of lumbar intervertebral discs. Acta Orthop Scand 46: 949-956.

Iozzo RV (1997) The family of the small leucine-rich proteoglycans: key regulators of matrix assembly and cellular growth. Crit Rev Biochem Mol Biol 32: 141-174.

Iozzo RV (1999) The biology of the small leucine-rich proteoglycans. Functional nework of interactive proteins. J Biol Chem 274: 18843-18846.

Kalamajski S, Oldberg Á (2007) Fibromodulin binds collagen type I via Glu-353 and Lys-355 in leucine-rich repeat 11. J Biol Chem 282: 26740-26745.

Kalamajski S, Oldberg Á (2010) The role of small leucine-rich proteoglycans in collagen fibrillogenesis. Matrix Biol 29: 248-253.

Kao WWY, Liu CY (2003) Roles of lumican and keratocan on corneal transparency. Glycoconjugate J 19: 275-285.

Kotwal R, Harris AM, Wright AE, Hodgson P, Hughes CE, Roberts S, Richardson J, Caterson B, Dent C (2007) Monoclonal antibodies recognising keratan sulphate (KS) proteoglycans, "KS-stubs" and KS sulphation motifs used in ELISA to quantify changes in proteoglycan metabolism in musculoskeletal diseases. J Bone Jt Surg 91-B: 292.

Liu CY, Birk DE, Hassell JR, Kane B, Kao WWY (2003) Keratocan-deficient mice display alteration in corneal structure. J Biol Chem 278: 21672-21677.

Marchand F, Ahmed AM (1990) Investigation of the laminate structure of lumbar disc annulus fibrosus. Spine 15: 402-410.

Masuda K, Lotz JC (2010) New challenges for intervertebral disc treatment using regenerative medicine. Tissue Eng 16: 147-158.

Melrose J, Ghosh P, Taylor TKF (2001) A comparative analysis of the differential spatial and temporal distributions of the large (aggrecan, versican) and small (decorin, biglycan, fibromodulin) proteoglycans of the intervertebral disc. J Anat 198: 3-15.

Melrose J, Smith S, Appleyard R, Little C (2008) Aggrecan, versican and type VI collagen are components of annular translamellar crossbridges in the intervertebral disc. Eur Spine J 17: 314-324.

Merline R, Schaefer RM, Schaefer L (2009) The matricellular functions of small leucine-rich proteoglycans (SLRPs). J Cell Commun Signal 3: 323-335.

Nerurkar NL, Elliot DM, Mauck RL (2010) Mechanical design criteria for intervertebral disc tissue engineering. $\mathrm{J}$ Biomech 43: 1017-1030.

O’Halloran DM, Pandit AA (2007) Tissue-engineering approach to regenerating the intervertebral disc. Tissue Eng 13: $1927-1954$.
Panattoni GL, Todros T (1989) Fetal motor activity and spine development. Panminerva Med 31: 183-186.

Peacock A (1951a) Observations on the pre-natal development of the intervertebral disc in man. J Anat 85: 260-274.

Peacock A (1951b) Observations on the post-natal structure of the intervertebral disc in man. J Anat $\mathbf{8 6}$ : 162-179.

Reed CC, Iozzo RV (2003) The role of decorin in collagen fibrillogenesis and skin homeostasis. Glycoconjugate J 19: 249-255.

Rees SG, Waggett AD, Kerr BC, Probert J, Gealy EC, Dent CM, Caterson B, Hughes CE (2009) Immunolocalisation and expression of keratocan in tendon. Osteoarthritis Cartilage 17: 276-279.

Roughley PJ, White RJ, Magny MC, Liu J, Pearce RH, Mort JS (1993) Non-proteoglycan forms of biglycan increase with age in human articular cartilage. Biochem J 295: 421-426.

Roughley PJ, White RJ, Sc-Szabó G, Mort J (1996) Changes with age in the structure of fibromodulin in human articular cartilage. Osteoarthritis Cartilage 4: 153-161.

Rufai A, Benjamin M, Ralphs JR (1995) The development of fibrocartilage in the rat intervertebral disc. Anat Embryol 192: 53-62.

Schaefer L, Iozzo RV (2008) Biological functions of the small leucine-rich proteoglycans: From genetics to signal transduction. J Biol Chem 283: 21305-21309.

Schollum ML, Robertson PA, Broom ND (2009) A microstructural investigation of intervertebral disc lamellar connectivity: detailed analysis of the translamellar bridges. J Anat 214: 805-816.

Smith LJ, Nerurkar NL, Choi KS, Harfe BD, Elliot DM (2011) Degeneration and regeneration of the intervertebral disc: lessons from development. Disease Model Mech 4: 31-41.

Svensson L, Aszódi A, Reinholt FP, Fässler R, Heinegård D, Oldberg A (1999) Fibromodulin-null mice have abnormal collagen fibrils, tissue organisation, and altered lumican deposition in tendon. J Biol Chem 274: 9636-9647.

Svensson L, Närlid I, Oldberg A (2000) Fibromodulin and lumican bind to the same region on collagen type I fibrils. FEBS Lett 470: 178-182.

Sztrolovics R, Alini M, Mort JS, Roughley PJ (1999) Age-related changes in fibromodulin and lumican in human intervertebral discs. Spine 24: 1765-1771.

Viola M, Bartolini B, Sonaggere M, Giudici C, Tenni R, Tira ME (2007) Fibromodulin interactions with type I and II collagens. Connect Tissue Res 48: 141-148.

Walmsley R (1953) The development and growth of the intervertebral disc. Edinburgh Med J 60: 341-365.

Young MF, By Y, Ameye L, Chen XD (2002) Biglycan knockout mice: new models for musculoskeletal diseases. Glycoconj J 19: 257-262.

Zheng Z, Nguyen C, Zhang X, Khorsani H, Wank JZ, Zara JN, Chu F, Yin W, Pang S, Le A, Ting K, Soo C (2011) Delayed wound closure in fibromodulin-deficient mice is associated with increased TGF- $\beta 3$ signalling. J Invest Dermatol 131: 769-778. 


\section{Discussion with Reviewers}

Reviewer I: Is there an IVD pathology in the FMOD-/mice line, and is the IVD collagen architecture disrupted in these mice?

Authors: In FMOD-/- mice virtually all collagen fibre bundles are disorganised and have an abnormal morphology. There is no reported IVD pathology in these animals; and as far as I am aware, the IVD of these animals has not yet been examined. Nonetheless, the tissue organisation of their tendons has been shown to be quite severely disrupted (Svensson et al., 1999, text reference). Since the annulus fibrosus of the IVD shares many structural and biochemical similarities with tendon, it is reasonable to assume that these animals may indeed have an underlying IVD pathology. It is certainly an important issue worth addressing in this $\mathrm{KO}$ model, and one that we hope to pursue.

Reviewer II: Could the authors confirm that the same disc level (L1-2) was examined consistently for all foetal stages?

Authors: We concentrated specifically on the L1-2 disc, whenever possible, to facilitate comparative analysis of specimens. In this regard, the lowermost pair of ribs within the section series were used as a reference point to establish the position of the last thoracic vertebrae and hence the subjacent first lumbar vertebrae. The only developmental stage, where it was sometimes difficult to identify and photograph the exact L1-2 spinal level was at E15, for the following reason: At the E15 stage, the notochord frequently becomes detached from the adjacent spinal tissue during wax sectioning, making it difficult to photograph both the notochord and its surrounding disc condensation in their correct anatomical positions and within the same plane of focus. In these instances, we might have photographed adjacent discs in thoracic or lower lumbar segments; however we would like to stress that no differences were observed in the patterns of birefringence or SLRP expression.

Reviewer II: During development, the notochord is encased in a fibrous sheath. Do you think the cage could arise from or be a remnant of this notochordal sheath?

Authors: We do, in fact, believe that the collagen cage that develops around the foetal nucleus derives in part from the fibrous sheath that surrounds the notochord. As the notochord expands into the surrounding cell condensations, its collagenous sheath bulges outwards and appears to thin towards the margins of the nucleus, separating the notochordal cells within, from the chondrocytes outside. As shown in our schematic (Fig. 6) the surrounding chondrocytes become reorganised concentrically around the bulging nucleus and then appear to co-operate in the organised deposition of collagen fibre bundles (which appear to be passed from cell to cell) around this structure. Thus the notochordal sheath not only appears to contribute to the most basal aspect of the cage, but may also provide a structural template for the coordinated deposition of reinforcing fibres by the surrounding chondrocytes.

Reviewer II: I am curious as to the authors' opinion regarding the development of the inner and outer AF regions - were you able to discern a clear boundary, or was it a seamless transition? Why do you think there is such a marked difference in SLRP expression between the inner and outer AF, in the context of collagen fibrillogenesis in particular?

Authors: The histology of the foetal disc frequently suggests an abrupt boundary between cartilaginous inner and fibroblastic outer annulus, often exaggerated by stains such as Alcian Blue, which selectively contrast the higher sulphated glycosaminoglycan content of the inner annulus. However, under the electron microscope one cannot identify a hard and fast boundary; rather a seamless transition, as you say, between the two tissues. The marked difference in SLRP expression between inner and outer annulus may relate to the fact that the inner annulus is developmentally more advanced, in terms of collagen fibrillogenesis, than the outer annulus, i.e., the chondrogenic ECM of the inner annulus is established in advance of the fibrous ECM of the outer annulus, whose cells must first become organised into oriented laminae before they begin secreting their oriented collagen. The increased expression of SLRPs within the inner annulus may also relate to their known role in modulating the activity of growth factors such as TGF $\beta$, which are important in driving chondrogenesis.

Reviewer II: Please comment on the value of SLRP knockout mice for informing more specifically the roles of the molecules in collagen fibrillogenesis during development.

Authors: SLRP knockout mice are important tools in the study of collagen fibrillogenesis. Surprisingly, however, they appear to be underused models for the study of intervertebral disc development and growth; particularly since they have significant relevance to the annulus fibrosus. The results of this study, coupled with previous observations in tendon (Svensson et al., 1999, text reference) suggest that there may be an interesting IVD phenotype in fibromodulin-null mice, but this remains to be investigated. It is important to recognise, of course, that a pathological phenotype may not necessarily be manifest in the IVD tissues of these animals as there may be functional compensation between SLRP members. 